\title{
Time selected multiple algorithms for reliable Fo tracking in difficult recording conditions
}

Philippe Martin

Univ Paris Diderot, Sorbonne Paris Cité, CLILLAC-ARP EA 3967

https://doi.org/10.36505/ExLing-2011/04/0023/000192

\begin{abstract}
Prosodic data mining in large spontaneous speech corpora often requires acoustic analysis of recordings done in poor conditions. The most detrimental pertain to the absence first harmonic in voiced segments and the presence of echo. In such cases it is very difficult to separate inter vocalic consonantal voicing from echo, or recover Fo from remaining harmonics. To address these problems, 10 different pitch tracking algorithms were implemented in the software program WinPitch, in order to allow an expert user, guided by an underlying displayed narrow band spectrogram, to select manually the appropriate process to deliver a satisfactory Fo curve for a selected time segment.
\end{abstract}

Key words: intonation, fundamental frequency, spontaneous speech, speech corpus

\section{Introduction}

The development of relatively large scale spontaneous speech corpora made possible new investigations in the domain of the interaction between syntax and prosody. With this new kind of data, predictions of wellestablished theoretical approaches such as autosegmental-metrical can now be confronted with more diverse data than the ones obtained through experimental procedures implying only read or elicited speech.

As most of the pitch curves obtained today use the speech analysis software Praat, problems occur frequently due to the use of a default fundamental frequency tracking algorithm based on a modified autocorrelation method (Boersma, 1993). Not only the frequency scale displayed under Praat lack details and is difficult to read, but the mediocre quality of speech recordings often found in the most interesting cases results in locally erroneous melodic curves, leading at times to misleading theoretical interpretation of the data.

To address this problem, no less than ten different algorithms have been implemented in the analysis software WinPitch, allowing the user to use them selectively on defined time segments, in order to obtain a resulting Fo curve exhibiting a satisfactory match with the first or second harmonic of an underlying displayed narrow band spectrogram. Although this appropriate algorithm manual selection can be time consuming and requires some user expertise in the field, it is possible with this implementation to obtain clean fundamental frequency curves from recordings whose poor quality would otherwise give locally or globally a large number of errors.

ExLing 2011: Proceedings of 4th Tutorial and Research Workshop on Experimental Linguistics, 25-27 May, Paris, France 


\section{Current enemies of Fo tracking}

Numerous errors in the Fo tracking process can occur when the hypotheses under which a particular analysis algorithm is designed are not fulfilled. Among the most common due to specific recording conditions we have:

1. Recording microphone with a poor low frequency response preventing the first voice harmonic to be present in the spectrum and making it difficult to recover when the analysed voiced speech segment has few or no harmonics (case of intervocalic stop consonants, of some nasals, etc.);

2. Presence of echo in the recording room, giving trailing frequencies in some part of the spectrum (depending of the room dimensions and wall or window material), easily confused with the first or second harmonic of voiced stops;

3. Use of Automatic Volume Control (AVC) distorting the speech sound dynamic, giving erroneous intensity readings (especially in voiced unvoiced and unvoiced voiced transitions) and possibly saturation the speech signal;

4. When AVC is not used, inappropriate recording input level, giving either a locally saturated speech signal (level to high), or conversely a low signal to noise ration when the input level is too low (distance between the microphone and the speaker to large);

5. Multiple sound sources due to background noise or multiple speaker overlapping segments;

6. Use of low bitrate in mp3, wma or other similar coding. Although the resulting auditory quality of these compression schemes can appear satisfactory, they sometimes give problematic results in case of Fo tracking analysis. The wav format should be used in all cases whenever possible;

Although some of these conditions detrimental to a reliable fundamental frequency analysis can be avoided, a compromise is frequently to be found between the interest of material recorded and its technical qualities. Nevertheless, some simple rules can prevent the data analysis to become an impossible challenge of prosodic analysis.

\section{Multiple Fo tracking strategies}

Experts in fundamental frequency tracking have shown for a long time that to date no single algorithm gives reliable results in all cases (Hess, 1983). For instance, in some occasions, an autocorrelation algorithm will give good results for fast Fo change whereas a spectral based analysis will fail, and conversely the autocorrelation will fail in voiced segments correctly processed with a Fourier analysis based algorithm.

To address these limitations, new Fo tracking algorithms have been added to WinPitch in order to offer more flexibility in selecting appropriate algorithms for difficult cases. Presently, these algorithms are: Spectral comb (Martin, 1981), Spectral brush (Martin, 2000), Cepstrum (Noll, 1964), AMDF (Ross et al. 1974), Standard Autocorrelation (Rabiner, 1977), Praat modified autocorrelation (Boersma, 1993) and Yin autocorrelation (2002). 
Furthermore, the user can manually 1) define pitch period directly on the speech wave displayed with an appropriate zoom scale; 2) apply the spectral comb method on manually selected specific harmonics and 3) force Fo to be zero for a selected segment. All methods use adjustable parameters, and pitch curves generated elsewhere with the Praat .pitch format can be imported in WinPitch and used as a supplementary method.

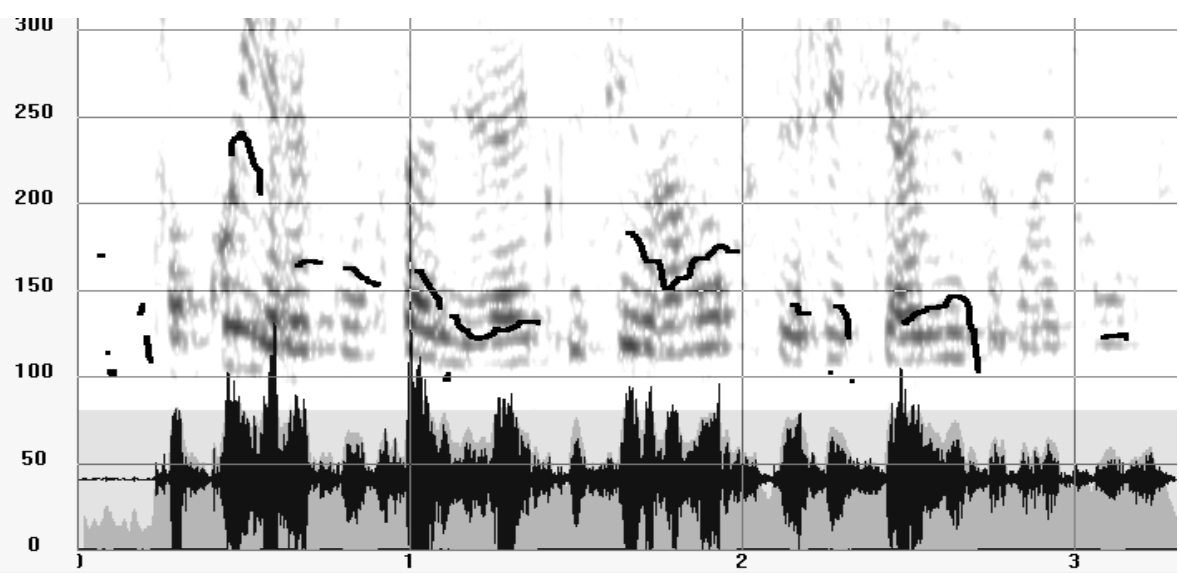

Figure 1. Rough pitch curve obtained by the spectral comb method. In this difficult example, the signal first harmonic is very weak and barely visible on the underlying narrow band spectrogram. Furthermore, the speech signal has been mp3 encoded with a very low bit rate (as used in inexpensive conference speech recorders).

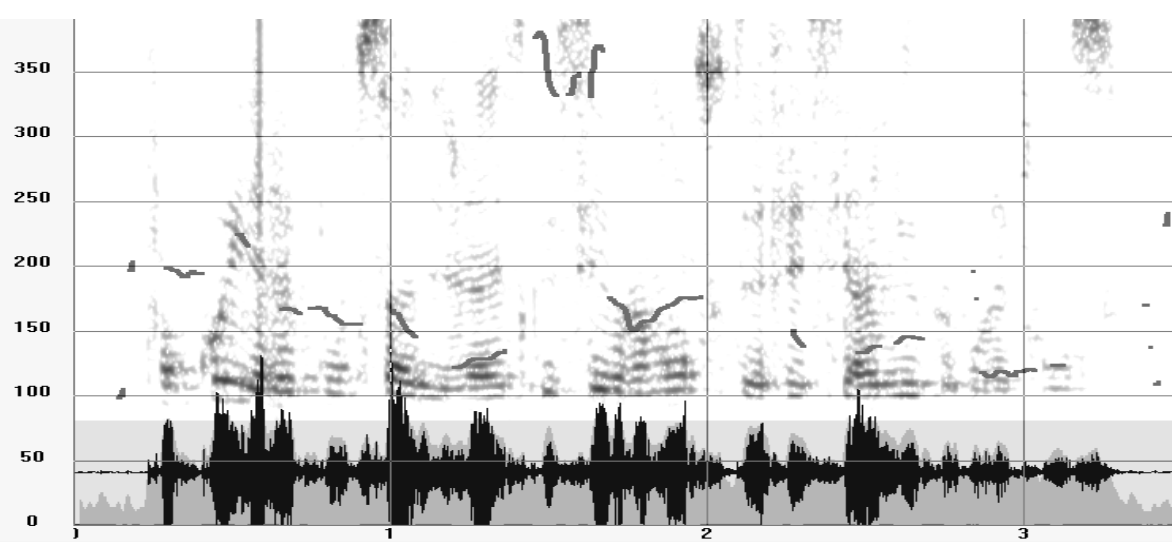

Figure 2. Pitch curve obtained by Praat default autocorrelation algorithm. In some section, the Fo value is either absent or erroneous.

This arrangement was first used on the Rhapsodie project to clean up Fo curves which were mostly tampered with many errors, preventing an 
automatic indexing labelling process at a later stage. Figures 3 gives an example of Fo correction using various algorithms, compared to the commonly used spectral comb (Fig. 1) and Praat pitch curve (Fig. 2).

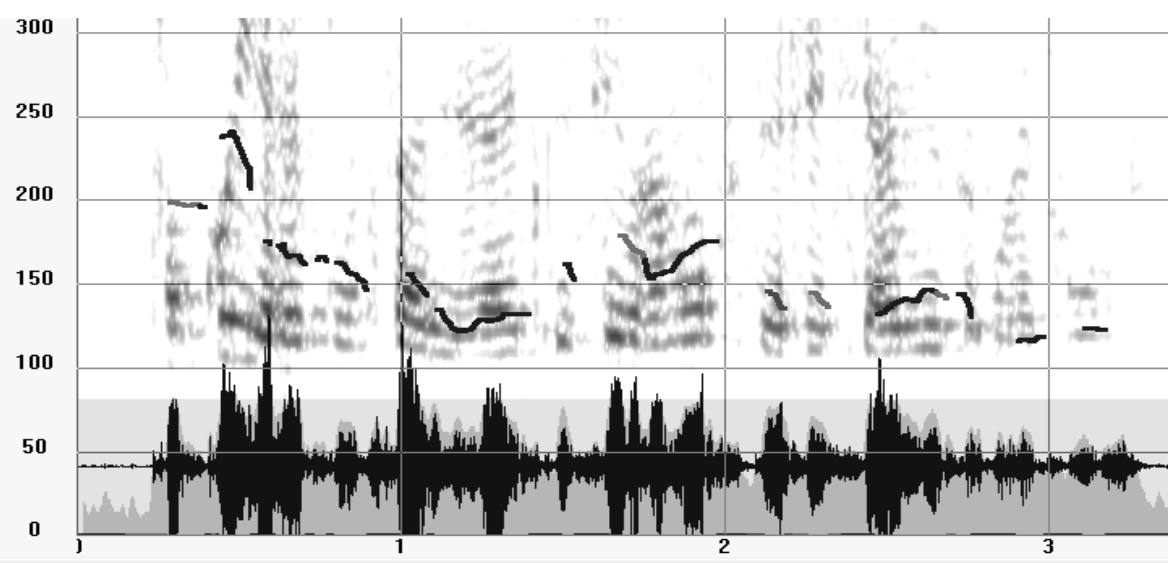

Figure 3. Corrected pitch curve using spectral comb on harmonic selection, AMDF and yin autocorrelation. Sections are displayed in different colours according to the method used locally.

\section{References}

Boersma, P. 1993. Accurate short-term analysis of the fundamental frequency and the harmonics-to-noise ratio of a sampled sound. Proc. of Institute of Phonetic Sciences of the University of Amsterdam, vol. 17, 97-110, Amsterdam, The Netherlands.

de Cheveigné, A., Kawahara, H. 2002. YIN, a fundamental frequency estimator for speech and music. JASA, 111:1917-1930.

Hess, W. 1983. Pitch Determination of Speech Signals: Algorithms and Devices. Heidelberg, Germany, Springer-Verlag.

Martin, Ph. 1981. Mesure de la fréquence fondamentale par intercorrélation avec une fonction peigne. Proc. 12th JEP, GALF, Montréal.

Martin, Ph. 2000. Peigne et brosse pour Fo : Mesure de la fréquence fondamentale par alignement de spectres séquentiels. Proc. of XXIIIèmes JEP Aussois, France.

Noll, A.M. 1964. Short-time spectrum and cepstrum techniques for vocal-pitch detection. JASA, vol. 36, 2, 296-302.

Rabiner, L.R. 1977. On the use of autocorrelation analysis for pitch detection. IEEE Trans. Acoust, Speech, Signal Processing, VOL. ASSP-25, NO. 1, 1977

Ross, M.J., Shaffer, H.L., Cohen, A., Freudberg, R., Manley, H. J. 1974. Average magnitude difference function pitch extractor. IEEE Trans. Acoust., Speech, Signal Processing, vol. ASSP-22, 353-362, Oct. 1974.

Praat: www.praat.org

Rhapsodie: Corpus de référence de français parlé, http://rhapsodie.risc.cnrs.fr/fr/

WinPitch: www.winpitch.com 